\title{
The Normative Data for Sensorineural Acuity Level (SAL) Test among Young Adults: Comparisons Between B71 and B81 Bone Transducers
}

\author{
Mahamad Almyzan Awang ${ }^{1}$, Ainul Hazwani Mohd Don ${ }^{1}$, Rosdan Salim², \\ Mohd Dasuki Sul'ain ${ }^{3}$ and Mohd Normani Zakaria, \\ ${ }^{1}$ Audiology Programme, School of Health Sciences, Universiti Sains Malaysia, Kubang Kerian, Kelantan, \\ Malaysia \\ ${ }^{2}$ Department of Otorhinolaryngology, School of Medical Sciences, Universiti Sains Malaysia, Kubang Kerian, \\ Kelantan, Malaysia \\ ${ }^{3}$ Biomedical Science Programme, School of Health Sciences, Universiti Sains Malaysia, Kubang Kerian, \\ Kelantan, Malaysia
}

\begin{abstract}
Introduction: The sensorineural acuity level (SAL) test is a valuable clinical assessment for hearing diagnosis, especially when the results of pure tone audiometry (PTA) are incomplete or questionable. Nevertheless, the literature on the SAL test is extremely limited and more research efforts are warranted to promote the application of this test in clinical settings. Essentially, the aim of the present study was to compare the normative data for the SAL test between two different bone vibrators (Radioear B71 and Radioear B81) among Malaysian participants.

Materials and Methods: Forty-two healthy Malaysian adults (aged 19 to 27 years) participated in this repeated-measures study. The PTA and SAL test was carried out according to the recommended protocols. The SAL normative data produced by the two bone transducers were then compared at different frequencies. Paired t-test and Bayesian inference were used for data analyses.

Results: The normative data for the SAL test gathered in the present study were descriptively higher than those reported among Caucasian adults. Relative to the Radioear B81 bone vibrator, the SAL normative data were significantly higher for the Radioear B71 bone transducer at all tested frequencies $\left(p<0.001, B F_{10} \geq 48.67\right)$.

Conclusions: The SAL normative data established by the two bone vibrators are statistically different. This information would be beneficial to provide clinicians with appropriate normative data when performing the SAL test on Malaysian patients.
\end{abstract}

Keywords: Sensorineural acuity level, Bone conduction, B81 bone transducer, Bayesian, Normative data.

\section{INTRODUCTION}

Blessed with two functioning ears, humans are able to enjoy everyday conversations, as well as to maintain body balance when pursuing routine activities [1]. If the hearing organs are affected due to specific causes, hearing loss and other related disorders would occur [1-5]. On the other hand, those with compromised balance organs would experience symptoms such as vertigo ("spinning sensation"), imbalance and other dizziness related problems [1, 6-]. As such, timely clinical management is imperative to minimize the negative consequences of these ear disorders [1, 911].

When sound waves are transmitted to the hearing organs, they are converted into nerve impulses and sent to higher parts of the brain for interpretation. In

*Address correspondence to this author at the Audiology Programme, School of Health Sciences, Universiti Sains Malaysia, 16150 Kubang Kerian, Kelantan, Malaysia; Tel: +609-7677691; Fax: +609-7677515;

E-mail: mdnorman@usm.my particular, the auditory system is divided into two parts: peripheral and central. The peripheral component consists of the outer ear, middle ear, inner ear, and auditory nerve. Whereas the central part of the auditory system is made of the brainstem and brain regions [1]. Clinically, there are three types of hearing loss, which is dependent on the site of the lesion. If the outer ear or middle ear is affected, conductive hearing loss $(\mathrm{CHL})$ is said to occur [1, 2]. On the other hand, sensorineural hearing loss $(\mathrm{SNHL})$ would occur if the inner ear or the central auditory part is disrupted [1,2]. In this regard, obtaining an accurate hearing diagnosis is crucial to proceed with appropriate treatment options [1].

In clinical settings, routine audiological tests are carried out to diagnose hearing. Pure tone audiometry (PTA), speech audiometry, tympanometry and acoustic reflex test are among the widely used hearing assessments [1, 12-14]. PTA has been regarded as the gold standard test for hearing diagnosis as it is able the measure the degree of hearing loss, as well as the type of hearing loss across speech frequencies [1, 2]. In this 
testing, by using headphones or insert earphones, air conduction $(\mathrm{AC})$ thresholds are obtained and represent the degree of hearing loss. Conversely, when a bone transducer is placed on the mastoid area, bone conduction $(\mathrm{BC})$ thresholds are recorded, which are essential to determine the type of hearing loss. All the hearing thresholds (that represent the lowest level of sound that a person can hear at specific frequencies) are plotted on a graph known as an audiogram [1]. In normal-hearing ears, $\mathrm{AC}$ and $\mathrm{BC}$ thresholds do not exceed $20 \mathrm{~dB} \mathrm{HL}$ [1]. If the $A C$ thresholds are more than $20 \mathrm{~dB} \mathrm{HL}$, hearing loss is indicated. On the other hand, in cases of $\mathrm{CHL}$, the $\mathrm{BC}$ thresholds are within the normal limit and the AC thresholds are abnormal, producing significant air-bone gaps (ABGs) [1, 2]. In SNHL cases, both $B C$ and $A C$ thresholds exceed the normal limit but with no $A B G s$. It is worth mentioning that objective hearing tests including auditory brainstem response, auditory steady-state response, cortical auditory evoked potential and others can also be conducted if the respective clinicians face difficulties in obtaining good cooperation from the patients (such as when testing children who are hyperactive) [15-17].

Since cross-hearing may occur during the PTA testing (i.e., sounds presented to one ear can actually be heard by the other ear), an essential procedure, known as masking, is typically carried out [1]. By presenting a masking noise to the non-test ear and a signal of interest to the test ear, valid PTA results will be obtained (as the cross-hearing problem is overcome). Nevertheless, applying the masking noise to the non-test ear may also create an unintended problem. In particular, when employing the $B C$ masking procedure on $\mathrm{CHL}$ patients with large $\mathrm{ABGs}$ in both ears, the masking noise can be too much and cause "over masking" $[1,18]$. Consequently, the exact BC thresholds cannot be established, and the hearing diagnosis is incomplete [18].

The sensorineural acuity level (SAL) test is a useful method to determine masked (exact) BC thresholds in cases of over masking $[18,19]$. It is a non-invasive technique that assesses the degree to which an AC pure-tone signal is masked by a noise signal delivered directly to the cochlea via the $\mathrm{BC}$ stimulation. To estimate the masked $B C$ thresholds in patients with hearing loss, the SAL normative data must be obtained first. The SAL test procedure begins after the conventional PTA testing is completed in normalhearing participants. The headphones are placed on both ears during the SAL test, while the bone vibrator is placed in the centre of the forehead. Narrowband noises are presented continuously at the maximum level through the bone vibrator. At the same time, the $A C$ threshold (in the presence of noise) is measured at frequencies of $250 \mathrm{~Hz}, 500 \mathrm{~Hz}, 1000 \mathrm{~Hz}, 2000 \mathrm{~Hz}$ and $4000 \mathrm{~Hz}$. At each frequency, the quantity of AC threshold shifts (differences between PTA in quiet and SAL test in noise) is computed. These values serve as the required SAL normative data $[18,19]$. It is worth stating that to estimate the exact $B C$ thresholds in over masking cases, the AC thresholds from both PTA and $S A L$ tests are required. The AC thresholds in the patient's SAL test will then be subtracted from the normative data obtained earlier in the SAL test. These values will represent the amount of estimated air-bone gaps (and estimated BC thresholds can be determined).

Even though having the SAL test can be advantageous in clinical settings, more research efforts are warranted to promote the use of this test among clinical professionals. Among others, establishing appropriate SAL normative data for a specific population is essential. Furthermore, it is also of interest to see whether the SAL normative are different when tested with different types of bone transducers. For many decades, Radioear B71 bone vibrator has been used widely in PTA testing [1, 2]. More recently, a newly designed bone transducer, Radioear B81, has been reported to be superior to the B71 bone vibrator (i.e., better acoustical characteristics) [2, 20, 21]. Essentially, the aim of the present study was to compare the normative data for SAL test between B71 and B81 bone vibrators among Malaysian adults. This information will be useful to provide clinicians with appropriate normative data when performing the SAL test on Malaysian patients.

\section{MATERIALS AND METHODS}

\section{Participants}

In the present study that employed a repeatedmeasures design, 42 young Malaysian adults (aged 19 to 27 years) were enrolled. They were chosen randomly among students and staff members of the respective institution. They were all in good health and had no hearing problems. As such, in the PTA testing, their hearing thresholds were equal to or less than 20 $\mathrm{dB} \mathrm{HL}$ at frequencies ranging from 250 to $8000 \mathrm{~Hz}$ bilaterally. Their ear canal was also clear with an intact and well-functioning tympanic membrane for both ears (as revealed by otoscopy and tympanometry). Prior to the data collection, ethical approval was obtained from 
the respective institutional review board (USM/JEPeM/18090408), which is in accordance with the 1975 Declaration of Helsinki and its subsequent amendments. All participants provided their consent forms indicating voluntary participation in the study.

\section{Test Procedure}

Using a calibrated two-channel audiometer (GSI 61, Grason-Stadler Inc., USA), all the participants underwent the PTA testing and followed by the SAL test procedure. Prior to the testing, proper instructions were given to the participants (i.e., they were required to push a response button whenever they heard the tone and release it when they no longer heard the tone). The PTA testing was carried out according to the established clinical protocol [1]. While wearing TDH-39 headphones, AC thresholds were measured at frequencies of $250 \mathrm{~Hz}, 500 \mathrm{~Hz}, 1000 \mathrm{~Hz}, 2000 \mathrm{~Hz}$, $4000 \mathrm{~Hz}$ and $8000 \mathrm{~Hz}$ for each ear (Figure 1). Subsequently, the $\mathrm{BC}$ testing was performed using the Radioear B71 bone vibrator placed on the mastoid process (Figure 2). The tested frequencies were 250 $\mathrm{Hz}, 500 \mathrm{~Hz}, 1000 \mathrm{~Hz}, 2000 \mathrm{~Hz}$, and $4000 \mathrm{~Hz}$. The BC test procedure was repeated but with the use of the Radioear B81 bone oscillator. Figure 3 shows the comparison of the two bone transducers.

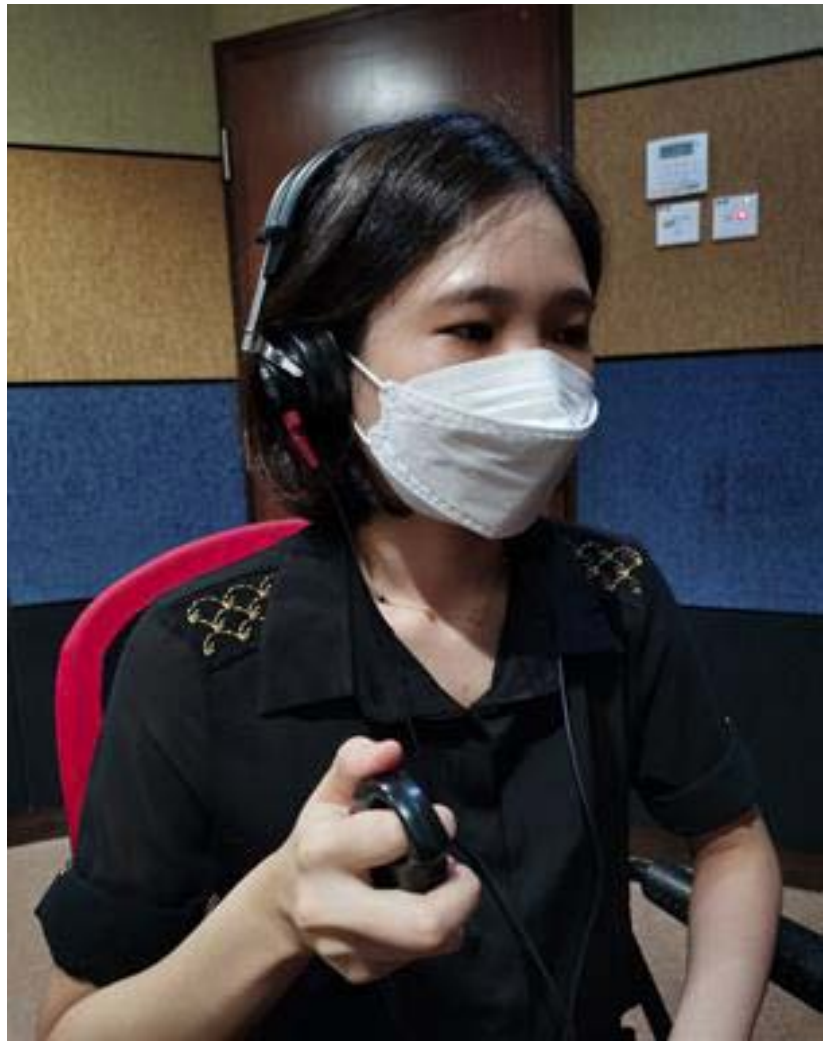

Figure 1: An example of air conduction testing in pure tone audiometry (PTA).

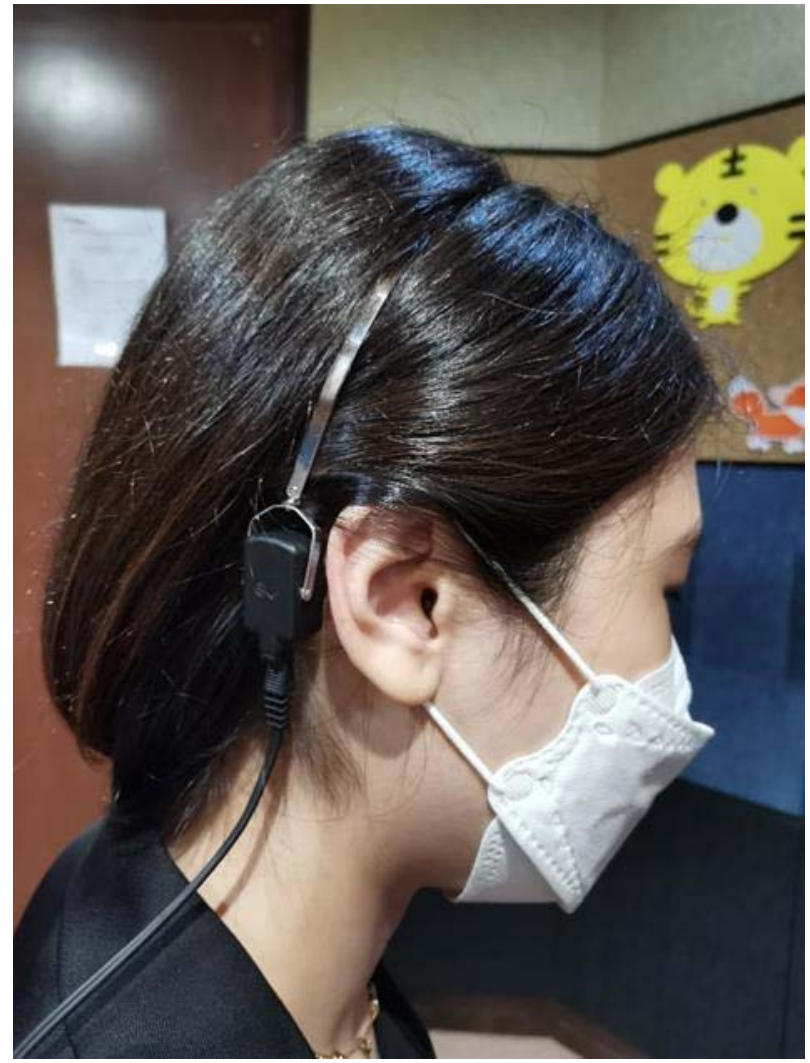

Figure 2: An example of bone conduction testing in pure tone audiometry (PTA).

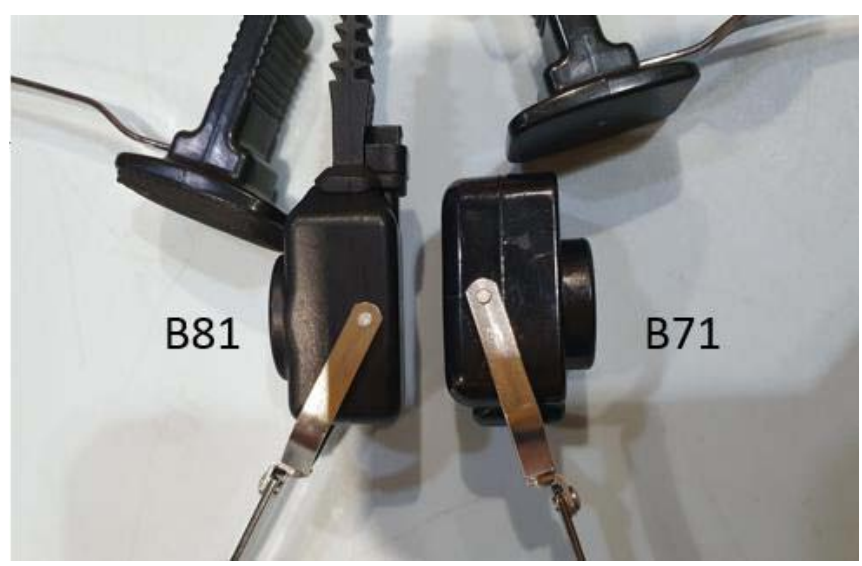

Figure 3: The exterior design of Radioear B71 and Radioear B81 bone transducers.

Upon the completion of the PTA testing, the SAL test was conducted according to the method established by Jerger and Tillman [19]. The method for this test was similar to the PTA, except that the bone vibrator was placed on the forehead with occlusion of both ears using the headphones (Figure 4). Narrowband noises were then presented at the maximum masking level continuously using the Radioear B71 bone conductor, while the pure tone was introduced to one ear via the headphones. The 
participants were instructed only to push the response button whenever they heard the tone (while ignoring the noise). The AC thresholds (in noise) were obtained for each ear across the frequencies of $250 \mathrm{~Hz}, 500 \mathrm{~Hz}$, $1000 \mathrm{~Hz}, 2000 \mathrm{~Hz}$, and $4000 \mathrm{~Hz}$. The SAL test procedure was repeated with the Radioear B81 bone transducer. The SAL normative data were computed as the differences between $A C$ thresholds in quiet (by PTA) and AC thresholds in noise (by SAL test). All the test procedures were carried out in a dedicated soundproof room within the Audiology Clinic, University Hospital.

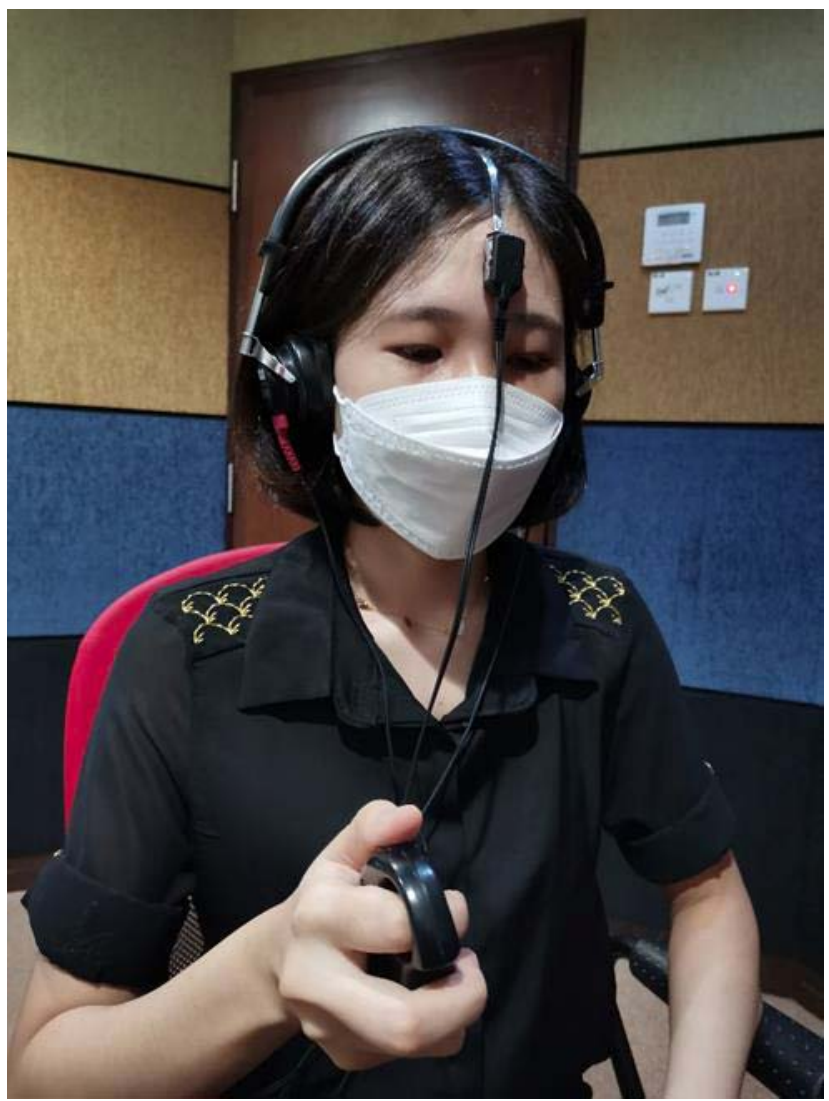

Figure 4: An example of sensorineural acuity level (SAL) test procedure.

\section{Statistical Analyses}

The obtained data were analysed using both descriptive and inferential statistics. As such, mean, standard deviation (SD) and percentage were expressed as applicable. The Shapiro-Wilk normality test found the data to be normally distributed, hence a paired t-test was used to compare the SAL normative data between left and right ears. The paired t-test was also employed to compare the SAL normative values between the two bone transducers at the specific frequencies. The statistical significance level was set at $p<0.05$. Additionally, the Bayesian version of the paired t-test was used to provide further evidence whether the data were in favour of the alternative hypothesis or the null hypothesis $[22,23]$. That is, the results do not favour either hypothesis if the Bayes factor, $\mathrm{BF}_{10}=1$. In terms of the strength of evidence, $\mathrm{BF}_{10}$ value between 1-3 indicates anecdotal evidence for the alternative hypothesis, 3-10 represents substantial evidence for the alternative hypothesis, 1030 implies strong evidence for the alternative hypothesis, 30-100 suggests very strong evidence for the alternative hypothesis and $\mathrm{BF}_{10}$ value of more than 100 indicates decisive evidence for the alternative hypothesis [22]. All data analyses were carried out using JASP statistical software (version 0.11.1, University of Amsterdam, Netherlands).

\section{RESULTS}

In the present study, the mean age of the participants was 22.9 years (SD $=1.3$ years). Of 42 , $62 \%(n=26)$ were females and the remaining $38 \%$ were males $(n=16)$. In terms of ethnicity, the majority of them were Malay (76\%), followed by Chinese (14\%), Indian (3\%) and other ethnic groups (7\%).

The SAL normative data had been obtained successfully from all participants. The paired t-test found the data between left and right ears to be not statistically different $(p>0.05)$. As such, the left and right data were then pooled for the subsequent analyses (a total of 84 ears).

Table 1 shows the normative data for SAL test for both bone transducers at specific frequencies $(n=84)$. The respective statistical analysis results are also revealed. As indicated, the normative values for the B71 bone vibrator are descriptive than those for the B81 bone transducer at all tested frequencies. This observation was then confirmed by the paired t-test results, in which significant differences were found in the SAL normative data between the two bone transducers at each frequency $(p<0.001)$, with effect size values, ranged from $0.40-0.88$. The smallest and largest effect sizes were noted at $2000 \mathrm{~Hz}(\mathrm{~d}=0.40)$ and $4000 \mathrm{~Hz}(\mathrm{~d}=0.88)$, respectively.

Figures $\mathbf{5}$ to $\mathbf{9}$ show the Bayesian inference results when the SAL normative data were compared between the B71 and B81 bone vibrators at the specific frequencies. Except at $2000 \mathrm{~Hz}$, the evidence for the alternative hypothesis was decisive $\left(\mathrm{BF}_{10}>100\right)$ at all frequencies. At $2000 \mathrm{~Hz}$ frequency, the $\mathrm{BF}_{10}$ value was 48.67 implying very strong evidence for the alternative hypothesis (Figure 8). 
Table 1: Mean and Standard Deviation (SD) of Normative Data for Sensorineural Acuity Level (SAL) Test at Specific Frequencies, and the Respective Statistical Test Results

\begin{tabular}{|c|c|c|c|c|}
\hline Frequency $(\mathrm{Hz})$ & Bone Vibrator & Mean $\pm S D(d B)$ & $P$ value & $\mathrm{BF}_{10}$ \\
\hline \multirow{2}{*}{250} & Radioear B71 & $46.3 \pm 8.2$ & \multirow{2}{*}{$<0.001$} & \multirow{2}{*}{$>100$} \\
\hline & Radioear B81 & $38.5 \pm 9.1$ & & \\
\hline \multirow{2}{*}{500} & Radioear B71 & $61.1 \pm 7.2$ & \multirow{2}{*}{$<0.001$} & \multirow{2}{*}{$>100$} \\
\hline & Radioear B81 & $51.7 \pm 8.1$ & & \\
\hline \multirow{2}{*}{1000} & Radioear B71 & $66.5 \pm 8.0$ & \multirow{2}{*}{$<0.001$} & \multirow{2}{*}{$>100$} \\
\hline & Radioear B81 & $59.4 \pm 7.2$ & & \\
\hline \multirow{2}{*}{2000} & Radioear B71 & $60.0 \pm 8.7$ & \multirow{2}{*}{$<0.001$} & \multirow{2}{*}{48.67} \\
\hline & Radioear B81 & $54.9 \pm 9.7$ & & \\
\hline \multirow{2}{*}{4000} & Radioear B71 & $58.4 \pm 9.8$ & \multirow{2}{*}{$<0.001$} & \multirow{2}{*}{$>100$} \\
\hline & Radioear B81 & $50.0 \pm 8.1$ & & \\
\hline
\end{tabular}

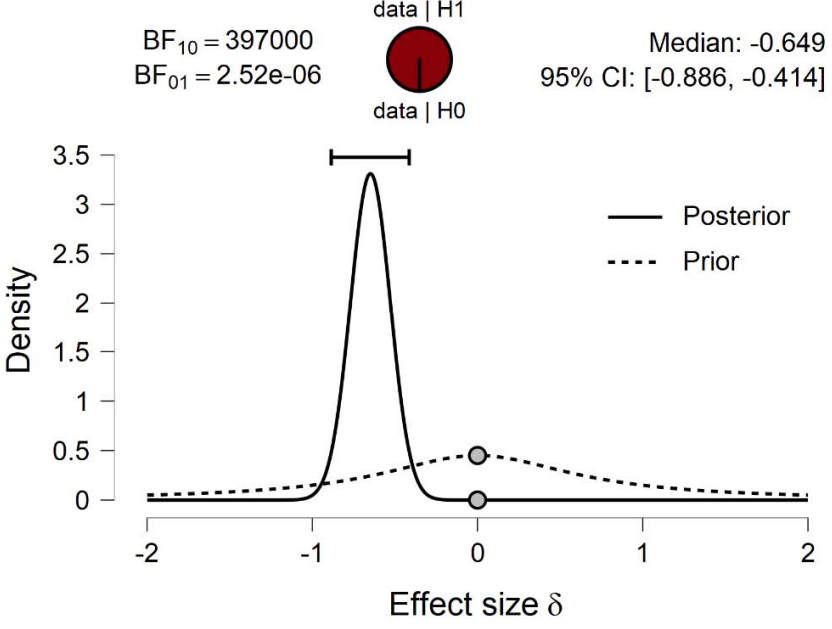

Figure 5: Bayesian statistical results when the SAL normative data at $250 \mathrm{~Hz}$ are compared between the two bone vibrators.

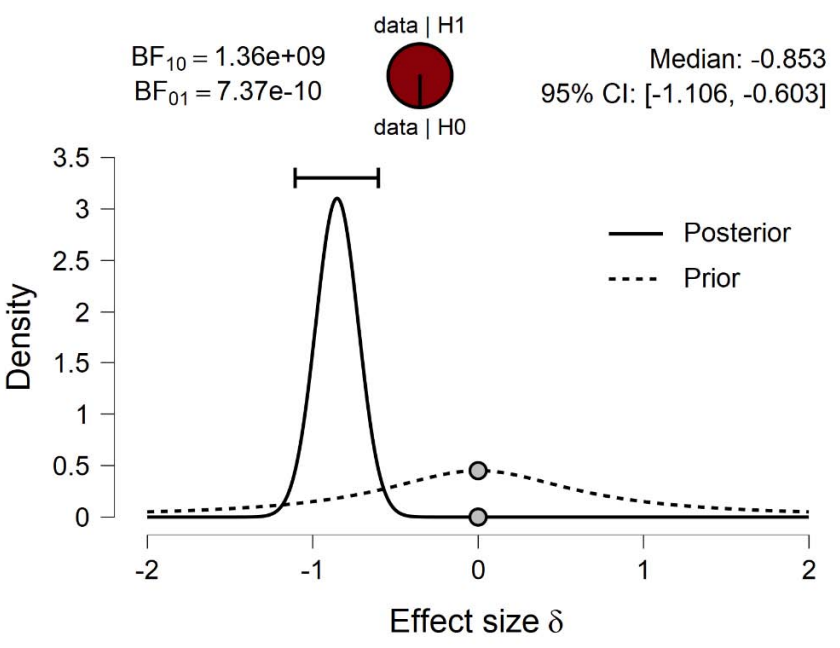

Figure 6: Bayesian statistical results when the SAL normative data at $500 \mathrm{~Hz}$ are compared between the two bone vibrators.

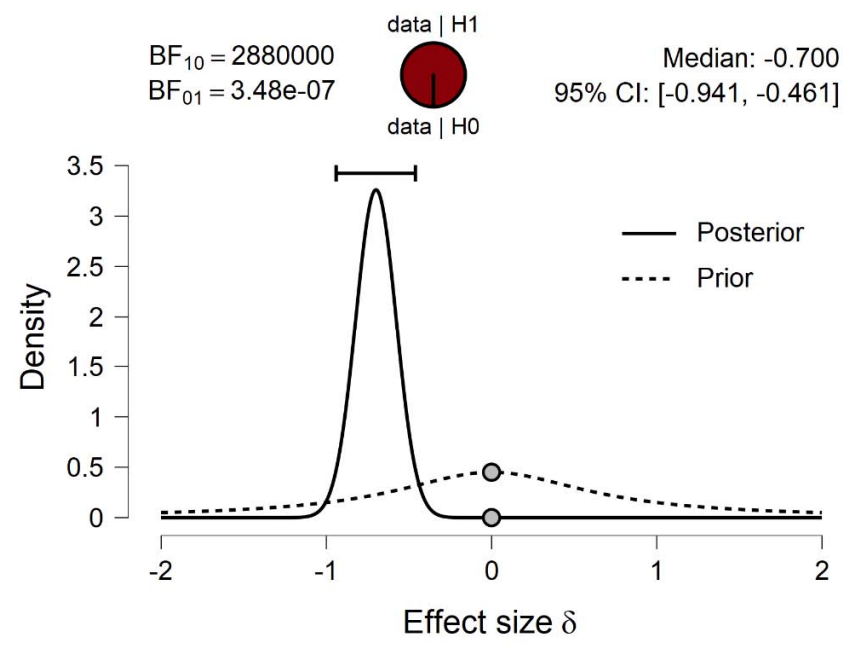

Figure 7: Bayesian statistical results when the SAL normative data at $1000 \mathrm{~Hz}$ are compared between the two bone vibrators.

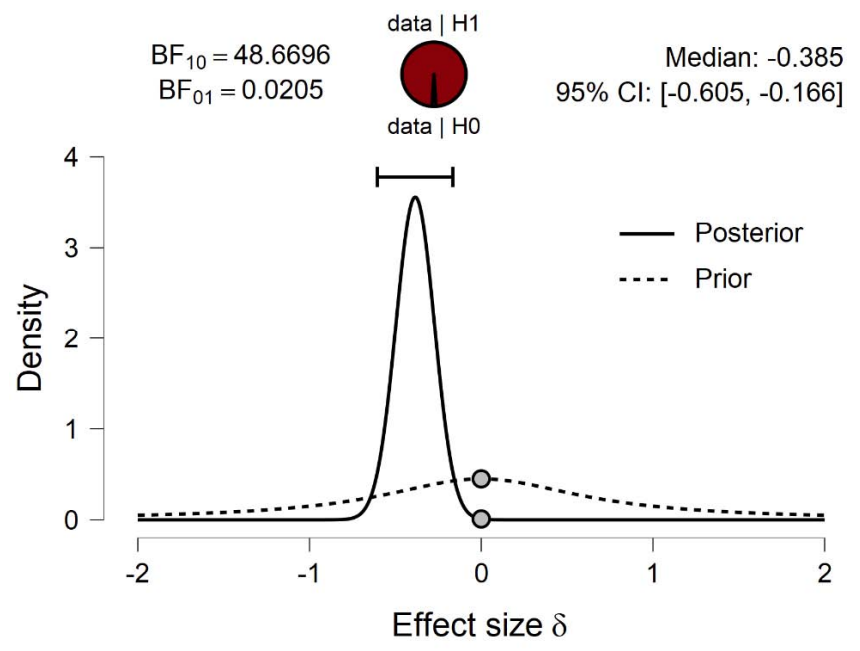

Figure 8: Bayesian statistical results when the SAL normative data at $2000 \mathrm{~Hz}$ are compared between the two bone vibrators. 


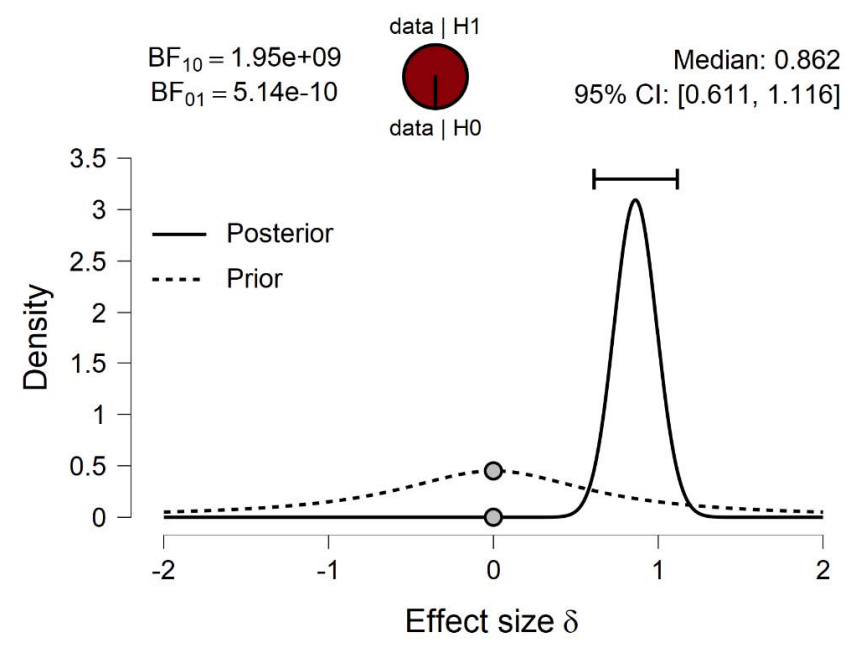

Figure 9: Bayesian statistical results when the SAL normative data at $4000 \mathrm{~Hz}$ are compared between the two bone vibrators.

\section{DISCUSSION}

Hypothesis testing utilizing the " $p$ value" is a common approach when analysing data in clinical studies [23, 24]. In particular, the obtained $p$ values are used to decide whether the null hypothesis is to be accepted or rejected. However, this frequentist inference has several limitations as the $p$ values are highly dependent on many critical factors (including the power of the study and the sample size) [23, 24]. Furthermore, smaller $p$ values do not indicate that the differences between the tested groups are larger (i.e., the magnitude of the difference is not provided). Optionally, Bayesian statistics has been introduced to provide alternative solutions in statistical decisions [22, 23]. In this analysis, rather than using the fixed criteria (i.e., if $p$ values are less than 0.05 , the null hypothesis is rejected or vice versa), it provides researchers with evidence (and strength of evidence) whether the study results are in favour of the alternative hypothesis or the null hypothesis. Several papers have been recently published highlighting the usefulness of the Bayesian statistics in audiological research $[25,26]$. In the present study, the statistical results provided by the $p$ value and Bayes factor $\left(\mathrm{BF}_{10}\right)$ were in fact consistent with each other, and appropriate statistical decisions could be made.

By definition, "normative data" refers to the establishment of the data as a baseline distribution from a reference population at a particular point or period for a score or measurement, and it is usually obtained from a large representative sample selected randomly from the general population [27]. In the present study, the normative data for the SAL test were established among healthy Malaysian adults $(n=84$ ears) using two types of bone transducers, i.e., B71 and B81. Of note, the literature on the SAL test is extremely limited (less than 10 published papers so far, even though it was first described in 1950s). In the study by Jerger and Tillman involving healthy Caucasian adults ( $n=48$ ears) [19], the SAL normative data were $20 \mathrm{~dB}$ at $250 \mathrm{~Hz}, 45 \mathrm{~dB}$ at $500 \mathrm{~Hz}$, and 50 $\mathrm{dB}$ at $1000 \mathrm{~Hz}, 2000 \mathrm{~Hz}$, and $4000 \mathrm{~Hz}$. Comparatively, the normative data for the SAL test established by the present study (by both transducers) are higher. Methodological differences (including ethnicity, different sample sizes and type of bone transducer) might have contributed to the discrepancy in the study outcomes. Generally, Caucasians have a bigger head size than Asians [28, 29]. Furthermore, the larger bone tends to have higher bone mineral density than the smaller bone due to genetics, skeletal size, the composition of body size, lifestyle, and social determinants [30]. In line with this, Tobias et al. [31] found that the bone mineral density was lower in Asians than in Caucasians. As reported by Helzner et al. [32], the lower bone mineral density was associated with a reduction of hearing sensitivity. Owing to this, differences in the SAL normative data (between Malaysians and Caucasians) would be somehow expected. It is worth noting that the type of bone oscillator was not stated in the study by Jerger and Tillman [19]. On the other hand, in a study by Kapoor et al. involving 50 normal-hearing Indian adults ( $n=100$ ears) aged 18 to 60 years old [33], the SAL normative data were measured using the Radioear B81 bone vibrator. The median SAL normative data were $40 \mathrm{~dB}$ at $250 \mathrm{~Hz}, 60 \mathrm{~dB}$ at $500 \mathrm{~Hz}$, $60 \mathrm{~dB}$ at $1000 \mathrm{~Hz}, 55 \mathrm{~dB}$ at $2000 \mathrm{~Hz}$, and $50 \mathrm{~dB}$ at $4000 \mathrm{~Hz}$. These data are indeed comparable with the present's study findings (for the B81 bone vibrator).

In clinical settings, the Radioear B71 is the most commonly used bone vibrator worldwide. However, the new bone vibrator, Radioear B81, has been gaining popularity and is likely to be extensively used in routine audiological practices in Malaysia. Therefore, the present study also aimed to compare the performance of the two bone vibrators in the SAL test. As found, the SAL normative data produced by the B71 bone oscillator were significantly higher than those of the B81 bone transducer at all tested frequencies. This finding was rather expected given the distinct acoustical characteristics posed by these bone vibrators. It has been demonstrated that the Radioear B71 has higher total harmonic distortions, especially at low frequencies than the Radioear B81 [20, 21]. In line 
with this, Jansson et al. [21] found the vibrotactile sensation produced by the two devices to be significantly different. The increased sensation of vibrotactile during the presentation of noise via the Radioear B71 might contribute to differences in the SAL normative data. Another probable explanation is that the maximum level of noise displayed by the Radioear B71 was greater than that of the Radioear B81. As a result, the high level of noise that reaches the cochlea potentially makes it difficult for an individual to hear the tone presented through the headphones, resulting in larger AC threshold shifts in the SAL normative data.

There were several limitations acknowledged in the present study. Firstly, the sample size was modest, and perhaps better study outcomes would be obtained if more samples are recruited. Nevertheless, it is worth noting that with the sample size of 84 subjects, the measured power of the study ranged from $99.7 \%$ to $100.0 \%$, indicating that the sample size is indeed sufficient to achieve the desired statistical outcomes. Secondly, this study only recruited normal-hearing subjects, and further research is warranted to verify the usefulness of the established SAL normative data (by both $B C$ transducers) when testing those with hearing loss.

\section{CONCLUSIONS}

Because masking problems in the PTA testing are common in clinical settings, the SAL test is a good option for achieving an accurate hearing diagnosis. In the present study, the normative data for the SAL test were compared between two bone vibrators. As reported, different bone transducers produce different SAL normative data, and this information is valuable to hearing health practitioners who wish to use SAL test in their clinical practice. Nevertheless, future research is welcome to ascertain the present study's findings.

\section{DISCLOSURE STATEMENT}

All authors declare that they have no conflicts of interest.

\section{FUNDING}

This study was funded by a Short-Term Grant (Universiti Sains Malaysia) (PO5040).

\section{REFERENCES}

[1] Katz J, Chasin M, English K, Hood L, Tillery KL. Handbook of clinical audiology. Philadelphia, PA: Lippincott Williams Wilkins, 2015.
[2] Wan Mohamad WN, Romli M, Awang MA, Abdullah R, Lih $A C$, Zakaria MN. The presence of unusual bone conduction thresholds in pure tone audiometry. Indian J of Otol 2020; 26(1): 54-57.

[3] Abdul Wahid SN, Md Daud MK, Sidek D, Abd Rahman N, Mansor S, Zakaria MN. The performance of distortion product otoacoustic emissions and automated auditory brainstem response in the same ear of the babies in neonatal unit. Int J Pediatr Otorhinolaryngol 2012; 76(9): 1366-1369. https://doi.org/10.1016/j.ijporl.2012.06.008

[4] Daud MK, Noh NF, Sidek DS, Abd Rahman N, Abd Rani N, Zakaria MN. Screening of dental staff nurses for noise induced hearing loss. B-ENT 2011; 7(4): 245-249.

[5] Zakaria MN, Nik Othman NA, Cheu Lih A. Tinnitus severity and the sound therapy outcome. Clin Exp Otorhinolaryngol 2015; 8(2): 179.

https://doi.org/10.3342/ceo.2015.8.2.179

[6] Zuraida Z, Mohd Normani Z, Dinsuhaimi S, Zalina I, Geshina MS. Development of Malay version of vertigo symptom scale (MVVSS) for clinical use. Malaysia J Med Health Sci 2010; 6(2): 31-39.

[7] Zainun Z, Zakaria MN, Sidek D, Ismail Z. Clinical use of Malay Version of Vertigo Symptom Scale (MVVSS) in patients with peripheral vestibular disorder (PVD). Med J Malaysia 2012; 67(4): 386-389.

[8] Zakaria MN, Tahir A, Zainun Z, Salim R, Mohd Sakeri NS Abdul Wahat $\mathrm{NH}$. The influence of type of visual image and gender on the perception of horizontality: a subjective visual horizontal (SVH) study. Acta Otolaryngol 2021; 141(1): 6265.

https://doi.org/10.1080/00016489.2020.1817552

[9] Aw CL, Tan SK, Abdullah R, Nik Othman NA, Zakaria MN Electrically evoked stapedial reflex threshold (ESRT) assessment among Malaysian cochlear implant users: Comparisons between different stimulus rates. Indian $\mathrm{J}$ Otol 2020; 26(2): 89-92.

[10] Zakaria MN. The effectiveness of sound therapy in treating patients with mild and severe tinnitus. Int $\mathrm{J}$ Disabil Hum Dev 2020; 19(2): 215-219.

[11] Husain WSW, Othman A, Othman NAN, Mohamad WNW Zakaria MN. Determining the internal and external reliability of depression, anxiety and stress scales (DASS-21) in assessing psychological symptoms among patients with tinnitus. NeuroQuantology 2018; 16(12): 97-102. https://doi.org/10.14704/nq.2018.16.12.1876

[12] Romli M, Wan Mohamad WN, Awang MA, Aw CL, Zakaria MN. The clinical value of bilateral bone conduction testing in hearing diagnosis. Indian J Otol 2020; 26(3): 182-185. https://doi.org/10.4103/indianjotol.INDIANJOTOL_106_20

[13] Lih AC, Zakaria MN, Mohamad RA, Nor Rashid MF. Effects of ethnicity and gender on the middle ear function in Asian adults. Indian J Otol 2017; 23: 94-97.

https://doi.org/10.4103/indianjotol.INDIANJOTOL $27 \quad 17$

[14] Awang MA, Zamri NN, Wan Mohamad WN, Zakaria MN. The influences of stimulation mode and stimulus frequency on acoustic reflex latency (ARL) among young adults. Int $\mathrm{J}$ Adolesc Med Health 2021; 33(4): 20180148. https://doi.org/10.1515/ijamh-2018-0148

[15] Zakaria MN, Jalaei B, Wahab NA. Gender and modulation frequency effects on auditory steady state response (ASSR) thresholds. Eur Arch Otorhinolaryngol 2016; 273(2): 349-354. https://doi.org/10.1007/s00405-015-3555-3

[16] Zakaria MN, Jalaei B, Aw CL, Sidek D. Are speech-evoked auditory brainstem response (speech-ABR) outcomes influenced by ethnicity? Neurol Sci 2016; 37(6): 943-948. https://doi.org/10.1007/s10072-016-2522-0

[17] Mukari SZS, Umat C, Chan SC, Ali A, Maamor N, Zakaria MN. Effects of age and type of stimulus on the cortical 
auditory evoked potential in healthy Malaysian children. J Audiol Otol 2020; 24(1): 35-39. https://doi.org/10.7874/jao.2019.00262

[18] Awang MA, Zakaria MN, Salim R, Rashid MFN. Overcoming masking problems in pure tone audiometry: Better understanding is needed regarding sensorineural acuity level (SAL) test. Int J Disabil Hum Dev 2021; 20(1): 21-25.

[19] Jerger J, Tillman T. A new method for the clinical determination of sensorineural acuity level (SAL). Arch Otolaryngol 1960; 71: 948-955. https://doi.org/10.1001/archotol.1960.03770060060008

[20] Eichenauer A, Dillon H, Clinch B, Loi T. Effect of boneconduction harmonic distortions on hearing thresholds. J Acoust Soc Am 2014; 136(2): EL96-102. https://doi.org/10.1121/1.4885771

[21] Jansson KJ, Håkansson B, Johannsen L, Tengstrand $T$. Electro-acoustic performance of the new bone vibrator radioear $\mathrm{B} 81: \mathrm{A}$ comparison with the conventional radioear B71. Int J Audiol 2015; 54: 334-340. https://doi.org/10.3109/14992027.2014.980521

[22] Jeffreys $H$. Theory of probability. Oxford (UK): Oxford University Press, 1961.

[23] Dienes Z. Using Bayes to get the most out of non-significant results. Front Psychol 2014; 5: 781. https://doi.org/10.3389/fpsyg.2014.00781

[24] Zakaria MN. The values of effect size in statistical decision for clinical research. Aud Vest Res 2017; 26: 1-3.

[25] McMillan GP, Cannon JB. Bayesian applications in auditory research. J Speech Lang Hear Res 2019; 62: 577-586. https://doi.org/10.1044/2018 JSLHR-H-ASTM-18-0228

[26] Zakaria MN, Tahir A, Zainun Z, Salim R, Mohd Sakeri NS, Abdul Wahat $\mathrm{NH}$. The influence of type of visual image and gender on the perception of horizontality: a subjective visual horizontal (SVH) study. Acta Otolaryngol 2021; 141(1): 6265

https://doi.org/10.1080/00016489.2020.1817552

[27] Anagnostou E, Mankad D, Diehl J, Lord C, Butler S, McDuffie A, et al. Normative data. In: Volkmar FR, ed. Encyclopedia of autism spectrum disorders. New York: Springer, 2013: 20622063.

[28] Ngeow WC, Aljunid ST. Craniofacial anthropometric norms of Malays. Singapore Med J 2009; 50: 525-528.

[29] Lih AC, Zakaria MN, Mohamad RA, Nor Rashid MF. Effects of ethnicity and gender on the middle ear function in Asian adults. Indian J Otol 2017; 23: 94-97. https://doi.org/10.4103/indianjotol.INDIANJOTOL $27 \quad 17$

[30] Leslie WD. Clinical review: Ethnic differences in bone massclinical implications. J Clin Endocrinol Metab 2012; 97(12): 4329-4340.

https://doi.org/10.1210/jc.2012-2863

[31] Tobias JH, Cook DG, Chambers TJ, Dalzell N. A comparison of bone mineral density between Caucasian, Asian and AfroCaribbean women. Clin Sci (Lond) 1994; 87(5): 587-591. https://doi.org/10.1042/cs0870587

[32] Helzner EP, Cauley JA, Pratt SR, Wisniewski SR, Talbott EO, Zmuda JM, et al. Hearing sensitivity and bone mineral density in older adults: the Health, Aging and Body Composition Study. Osteoporos Int 2005; 16(12): 1675-1682. https://doi.org/10.1007/s00198-005-1902-8

[33] Kapoor P, Zangmo N, Garg LN, Saini A, Gupta M. Threshold shift validity by documenting sensorineural acuity level: a useful tool for masking. Int J Otorhinolaryngol Head Neck Surg 2020; 6(5): 913-917. https://doi.org/10.18203/issn.2454-5929.ijohns20201686

https://doi.org/10.6000/1929-6029.2021.10.15

(C) 2021 Awang et al.; Licensee Lifescience Global.

This is an open access article licensed under the terms of the Creative Commons Attribution License (http://creativecommons.org/licenses/by/4.0/) which permits unrestricted use, distribution and reproduction in any medium, provided the work is properly cited. 